\title{
Beeindruckende Mathematik-Erfolge im 53. Bundeswettbewerb ,Jugend forscht
}

\author{
Jonas Bayer, Marco David, Simon Dubischar, Malte Haßler, \\ Abhik Pal, Michael Schmalian und Benedikt Stock \\ Zusammengestellt von Stephanie Schiemann
}

\begin{abstract}
In diesem Jahr gab es beim Bundeswettbewerb „Jugend forscht“ mehrere ausgezeichnete Arbeiten im Bereich der Mathematik. Die Fachgebietsjury mit Jürgen Richter-Gebert als Sprecher war fasziniert von der Qualität der diesjährigen Beiträge. Michael Röckner (Präsident der DMV): „Wegen der außergewöhnlich exzellenten

Arbeiten im Bereich der Mathematik entschloss sich die DMV kurzfristig, anstelle des einen üblichen

Sonderpreises für eine außergewöhnliche mathematische Arbeit, gleich drei zu vergeben. Diese drei ,Jugend-forscht'-Projekte, die sich hier vorstellen, haben im Fachgebiet Mathematik/Informatik den 2. und

3. Preis erzielt sowie den „Preis der Preise“, den fächerübergreifenden Bundessieger-Preis des

Bundespräsidenten. Wir hoffen, dass es auch in Zukunft wieder mehr starke Mathematik-Arbeiten geben wird. Ich möchte darauf hinweisen, dass diese drei Arbeiten von zwei engagierten Professoren betreut wurden, die auch in früheren Jahren mehrfach auf dem Bundeswettbewerb prämierte Arbeiten betreut hatten:

Frank Herrlich aus Karlsruhe und Dierk Schleicher aus Bremen. Deutschlandweit wird es vermutlich noch weit mehr Potential für erfolgreiche Nachwuchsarbeiten geben. Dieser Artikel soll Kolleginnen und Kollegen an Universitäten und Schulen anregen, sich hier unterstützend zu engagieren."
\end{abstract}

Aus Bremen kommen gleich zwei erfolgreiche „Jugend forscht"-Projekte, die von Dierk Schleicher (Bremen) gemeinsam mit Yuri Matiyasevich (Sankt Petersburg) betreut wurden:

Hilbert meets Isabelle, eingereicht von Marco David (17), Benedikt Stock (19), Abhik Pal (19). Alle drei sind Mathematikstudenten der Jacobs University Bremen.

Sie erhielten den Preis für eine außergewöhnliche Arbeit - Bundessieg (gestiftet vom Bundespräsidenten Frank-Walter Steinmeier) und zusätzlich die Einladung zum 30. European Union Contest for Young Scientists in Dublin, Irland (gestiftet von der Europäischen Kommission), den Europa-Preis für Teilnehmer am European Union Contest for Young Scientists in Dublin, Irland (gestiftet von der Deutschen Forschungsgemeinschaft) und den

Von (Hilbert, Isabelle) und anderen universellen Paaren

Wir kamen gerade aus einer der größten Kunstausstellungen der Welt, der Eremitage in St. Petersburg. Auf dem Vorplatz Dierk Schleicher, der uns jemanden vorstellen wollte: Yuri Matiyasevich - der russische Mathematiker, der 1970 das 10 . Hilbertsche Problem beantworten konnte. Damals ahnte wohl noch niemand, dass wir zwei Jahre später wieder nach St. Petersburg fahren würden, diesmal auf Einladung von Matiyasevich, um ihm die Ergebnisse unserer Forschung zu präsentieren.

Nach diesem ersten Treffen schauten wir uns Matiyasevichs Mathematik genauer an. Um die Motivation und
Preis für eine außergewöhnliche mathematische Arbeit (gestiftet von der DMV).

Optimierung diophantischer Gleichungen, eingereicht von Simon Dubischar (17), Schüler des Kippenberg Gymnasium Bremen im Abiturjahrgang 2019, Jonas Bayer (18), Malte Haßler (18), beide Mathematikstudenten der Jacobs University in Bremen.

Sie erhielten den 3. Preis im Fachgebiet Mathematik/Informatik (gestiftet von der Fraunhofer-Gesellschaft zur Förderung der angewandten Forschung e. V.), zusätzlich den Preis für eine außergewöhnliche mathematische Arbeit (gestiftet von der DMV). Außerdem wurden sie von der DMV als "Mathemacher des Monats Juli 2018“ ausgewählt.

Hintergründe besser zu verstehen, trafen wir uns im Herbst 2016 gemeinsam mit Dierk zu einem MathematikWochenende. Dort kehrten wir zum Ursprung des Problems zurück, der im Beginn des letzten Jahrhunderts liegt.

Das zehnte Hilbertsche Problem Im Jahr 1900 stellte der Mathematiker David Hilbert auf dem Internationalen Mathematikkongress in Paris 23 ungelöste Probleme vor, die die Mathematik des kommenden Jahrhunderts bestimmen sollten. Das 1o. Hilbertsche 
Problem beschäftigt sich mit der Lösbarkeit von diophantischen Gleichungen. Beispiele für diophantische Gleichungen sind $a^{2}+b^{2}=c^{2}$ oder $3 x-5=4$, Polynomgleichungen mit ganzzahligen Koeffizienten. Hilbert forderte, „man solle ein Verfahren angeben, mit dem sich bei einer jeden diophantischen Gleichung entscheiden lässt, ob sie eine Lösung besitzt".

Dabei sind allerdings nur Lösungen in den natürlichen Zahlen gemeint. So hat beispielsweise die Gleichung $3 x-5=4$ die Lösung $x=3$. Die Gleichung $x^{2}=2$ hätte allerdings keine Lösung, da die Wurzel von 2 keine natürliche Zahl ist. Nun stellten sich die Mathematiker des 20. Jahrhunderts aber die Frage: Gibt es überhaupt ein Computerprogramm, das für jede diophantische Gleichung sagen kann, ob sie lösbar ist?

Nach langer Vorarbeit von Martin Davis, Julia Robinson und Hilary Putnam konnte Yuri Matiyasevich schließlich beweisen, dass die Antwort „Nein“ lautet: Ein solches Programm, das heißt einen Algorithmus, kann es nicht geben. Je mehr wir uns mit der Thematik beschäftigten, desto mehr erkannten wir, wie weitreichend diese Aussage ist und in wie vielen mathematischen Bereichen sie sich anwenden lässt. Schließlich beschlossen Simon, Malte und Jonas ein eigenes Forschungsprojekt zu starten und nahmen gemeinsam 2017 an ,Jugend forscht teil.

\section{Ein Hilbert-Problem - Zwei Projekte}

Nachdem wir mit Matiyasevich in Kontakt getreten waren und ihm von unserem Projekt berichtet hatten, besuchte er uns letzten Herbst in Bremen - mit der nächsten Projektidee im Gepäck. Sein Vorschlag war es, die Methoden des 21. Jahrhunderts auf seine Mathematik des 20. Jahrhunderts anzuwenden, denn sein Beweis ist sehr komplex und nicht einfach von Menschen zu überprüfen. Mittlerweile gibt es jedoch die Möglichkeit, eine formale Verifikation mithilfe des Computers durchzuführen. Dazu schreibt man den Beweis formal auf, sodass er von einem Computer Schritt für Schritt nachvollzogen werden kann. Einige Studierende der Jacobs University Bremen darunter auch Benedikt und Marco starteten das Formalisierungsprojekt.

Gegen Ende seines Besuches in Bremen stellten wir Matiyasevich außerdem die Frage, welche weitere Forschung zu Hilberts 10. Problem betrieben werden könnte. In unserer bisherigen Forschung hatten wir festgestellt, dass diophantische Gleichungen auch sehr komplex und lang werden können - mit tausenden von Variablen. Matiyasevich erläuterte uns, dass man Abschätzungen machen kann, wie komplex diese Gleichungen werden. Schließlich setzten sich Simon, Malte und Jonas an das Komplexitäts-Projekt.

\section{Das Formalisierungsprojekt - Hilbert meets Isabelle} Für die Formalisierung empfahl Matiyasevich uns das interaktive Beweissystem Isabelle/HOL. Dieses wird in Zusammenarbeit der TU München und der Cambridge University entwickelt. Zum Zeitpunkt des Schreibens waren weite Teile des Beweises bereits formalisiert und von Isabelle bestätigt worden. Um einen besseren Über- blick über den bisherigen Stand zu geben, möchten wir zunächst näher auf den Beweis eingehen und die Grundideen erklären.

Matiyasevichs Beweis lässt sich grob in zwei Teile unterteilen: Der erste Teil enthält Matiyasevichs wichtige neue Erkenntnis, mit Hilfe derer er die jahrzehntelange Vorarbeit von anderen Mathematikern vervollständigen konnte. Er bewies, dass sich Potenzieren diophantisch ausdrücken lässt, es also ein Polynom $P\left(a, b, c, x_{1}, \ldots x_{n}\right)$ gibt, mit der Eigenschaft, dass für gegebene $a, b, c$ es genau dann natürliche Zahlen $x_{1}, \ldots, x_{n}$ gibt, sodass das Polynom gleich null ist, wenn $a=b^{c}$ gilt.

Dieses Ergebnis wird im zweiten Teil verwendet. Dort wird eine sogenannte Registermaschine eingeführt. Eine Registermaschine ist ein Modell für einen Computer, das äquivalent zur bekannteren Turing Maschine ist. Mengen, die von solchen Computern akzeptiert werden können (d.h. die Maschine hält genau für Elemente der Menge als Eingabe), heißen rekursiv aufzählbar. Diese Maschine kann durch mathematische Gleichungen simuliert werden, welche "fast" diophantisch sind, allerdings Potenzieren benötigen. Da sich nach dem ersten Teil aber auch das Potenzieren diophantisch ausdrücken lässt, kann jeder Schritt der Registermaschine diophantisch beschrieben werden. Folglich können wir jede rekursiv aufzählbare Menge diophantisch beschreiben. Durch einfaches Ausprobieren erkennt man leicht, dass jede diophantische Menge rekursiv aufzählbar ist, d.h. die Konzepte „diophantisch" und „rekursiv aufzählbar" sind äquivalent.

Aus heutiger Sicht fragte Hilbert allerdings nicht nach rekursiver Aufzählbarkeit, sondern nach Entscheidbarkeit diophantischer Mengen, also nach einer "Ja/Nein“Antwort. Eine solche kann der Computer für rekursiv aufzählbare Mengen per Definition aber nicht unbedingt geben: Wenn die untersuchte Zahl nach einem Jahr Laufzeit noch nicht ausgegeben wurde, wird sie es vielleicht am Tag darauf oder sie wird niemals ausgegeben, man kann es nicht wissen. Da es rekursiv aufzählbare Mengen gibt, die nicht entscheidbar sind (Folge aus dem Halteproblem), gibt es auch diophantische Mengen, die unentscheidbar sind. Folglich kann der geforderte Algorithmus von Hilbert nicht existieren.

Bei unserer Arbeit konnte bisher der erste Teil, also die fundamental neue Arbeit von Matiyasevich in Gänze formalisiert und von Isabelle bestätigt werden. Beim zweiten Teil sind momentan Anfang und Ende des Beweises fertig, allerdings müssen einige der Theoreme noch verifiziert werden, um Hilbert und Isabelle zusammenzubringen.

Die Formalisierung wird nach Vollendung der gesamten Isabelle Community zur Verfügung gestellt, so dass jeder in Zukunft darauf zugreifen kann. Daneben zeigt unsere Arbeit, wie ausgeklügelt die Beweissysteme bereits sind und dass sie auch von Nicht-Experten bedient werden können. Vielleicht steuern wir auf eine Zukunft zu, in der jeder mathematische Beweis mit Computerhilfe verifiziert werden wird. Auf diese Weise können unwichtige Details vernachlässigt werden und Mathematiker können sich auf das Wesentliche, die Ideen in ihren Beweisen, konzentrieren. 


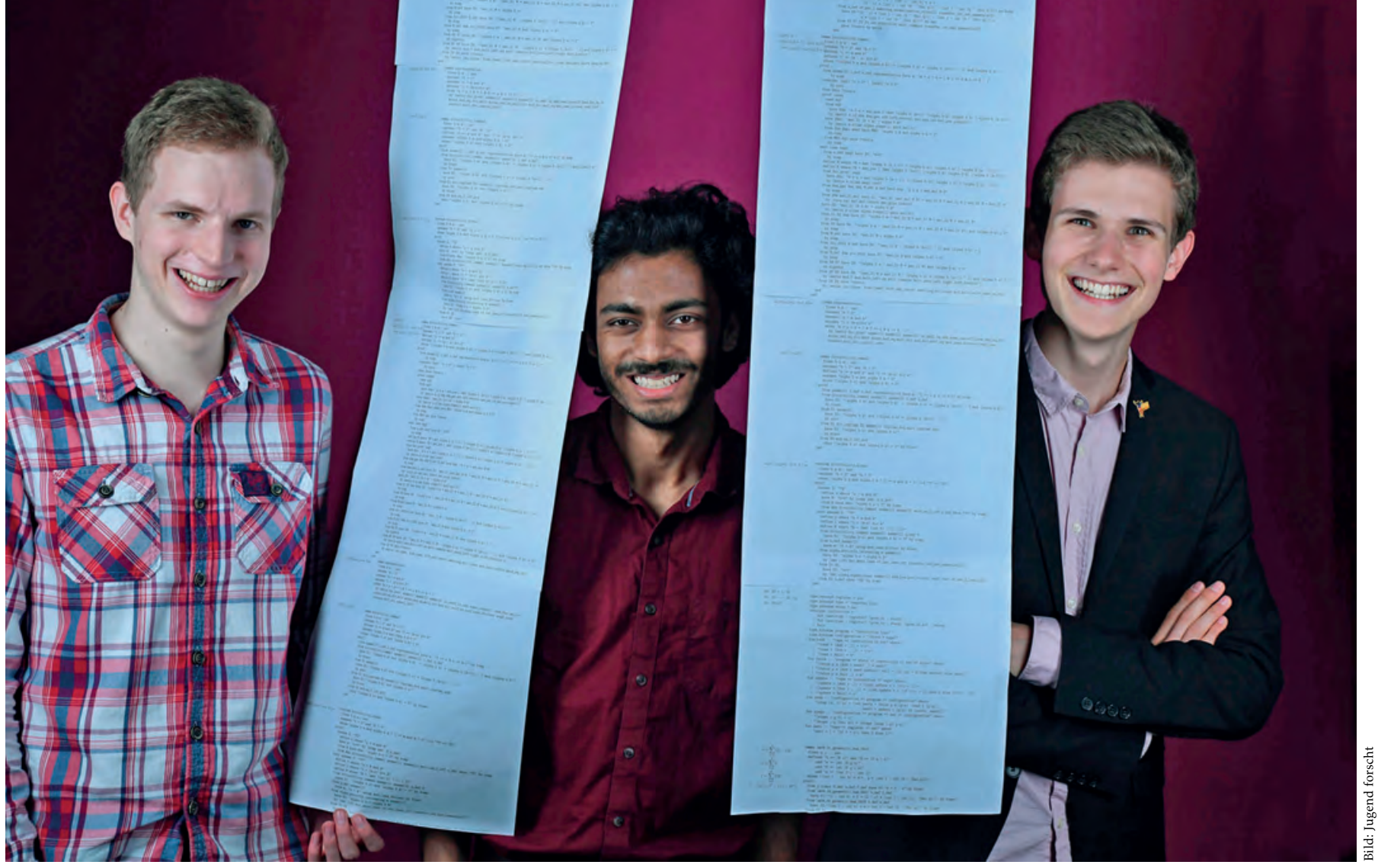

Projektbild des Formalisierungsprojekt Hilbert meets Isabelle: Benedikt Stock, Abhik Pal und Marco David (v. 1.n. r.)

Dass Computer auch Fehler finden können, die Menschen jahrelang übersehen haben, demonstriert eindrucksvoll Gödels Originalform seines ontologischen Gottesbeweises. Isabelle konnte nämlich in Gödels Axiomen, auf denen sein Beweis beruht, einen Fehler finden, der das gesamte System inkonsistent werden lässt. Etwas später konnte dann ein alternativer Beweis von Isabelle bestätigt werden. Weitere bekannte Beispiele von formal verifizierten Beweisen sind der Beweis der Keplerschen Vermutung unter Tom Hales sowie des 4-Farben-Satzes unter Georges Gonthier.

\section{Das Komplexitätsprojekt-Optimierung diophantischer Gleichungen}

Verschiedenste mathematische Fragestellungen lassen sich durch diophantische Gleichung und ihre Lösungsmengen ausdrücken. Daher stellten wir uns die Frage, wie komplex diese Gleichungen werden müssen, wenn die Probleme, die sie beschreiben, komplizierter werden. Als Maß für die Komplexität einer Gleichung führten Mathematiker das Konzept der universellen Paare ein. Ein Paar $(v, d)$ wird universell genannt, wenn jede beliebige diophantische Menge durch eine Gleichung mit $v$ Variablen und Grad $d$ beschrieben werden kann. Mathematiker konnten bisher schon einige solche Paare bestimmen, beispielsweise ist $(58,4)$ ein universelles Paar. Das Erstaunliche dabei: Jede noch so schwierige Fragestellung, die man überhaupt mit diophantischen Gleichungen ausdrücken kann, kann man auch mit einer Gleichung in nur 58 Variablen mit Grad 4 ausdrücken.
Die bisherigen Ergebnisse bezogen sich jedoch nur auf natürliche Zahlen, weshalb wir in unserem Projekt die vorhandene Theorie auf die ganzen Zahlen erweiterten. Eine einfache Methode dafür verwendet das Gauß-Lagrange Theorem, das besagt, dass jede natürliche Zahl durch drei ganze Zahlen $x, y, z$ in der Form $x^{2}+y^{2}+z^{2}+z$ dargestellt werden kann. Damit lassen sich die natürlichen Zahlen in jedem Polynom substituieren, wobei sich die Variablenanzahl dann verdreifacht und der Grad verdoppelt. Zum Beispiel resultiert aus dem universellen Paar $(58,4)$ das Paar $(174,8)$, wenn ganze Zahlen verwendet werden.

Durch aufwendigere Methoden lassen sich jedoch bessere universelle Paare finden. Ausgangspunkt waren die schon existenten universellen Paare in den natürlichen Zahlen und Ergebnisse des chinesischen Zahlentheoretikers Zhi-Wei Sun „Further Results On Hilbert's Tenth Problem“, in dem hergeleitet wird, dass jede beliebige diophantische Menge durch ein Polynom in 11 ganzzahligen Variablen darstellbar ist. Mit Hilfe eines Computer-Algebra-Systems konnten wir dann ausgehend von diesem Text beweisen, dass das Paar (11, 81141759977754190913401544094085693359375483328) für ganzzahlige Variablen universell ist.

Dabei ist der Grad jedoch sehr hoch, deshalb fragten wir uns, wie man durch Hinzufügen von nur wenigen Variablen den Grad möglichst stark reduzieren kann. Auch hierfür waren die bereits bestehenden Ergebnisse hilfreich. Wir konnten entsprechende Lemmas auch auf ganze Zahlen anwenden und dadurch bestimmte Terme mit besonders hohem Exponenten geschickt substituie- 


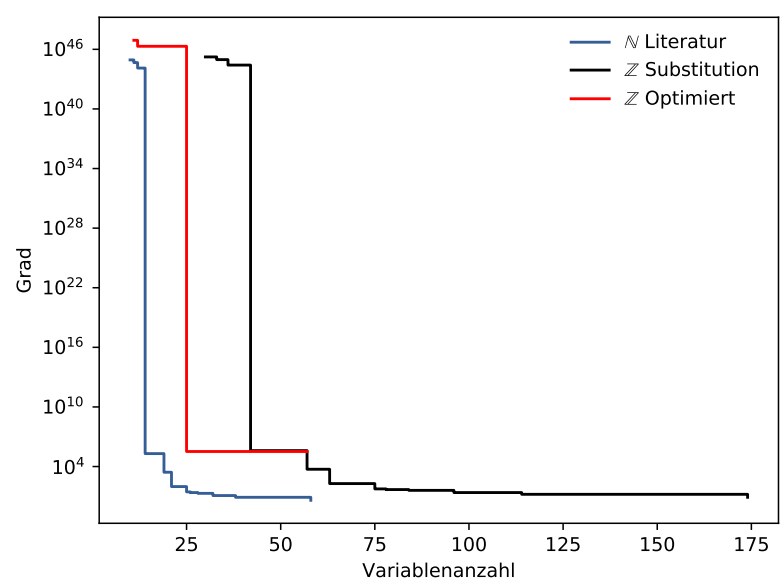

Die blaue Linie zeigt universelle Paare in den natürlichen Zahlen, wie sie bereits erforscht waren. Daraus ergibt sich die schwarze Linie für die ganzen Zahlen durch triviale Substitution. Unsere Optimierung für die ganzen Zahlen wird durch die rote Linie dargestellt.

ren. Somit konnte der Grad erheblich reduziert werden und wir erhielten u. a. die kompakten universellen Paare $(26,657680)$ und $(29,10028)$. Dieser Fortschritt ist auch im Diagramm zu sehen.

Epilog

Im Juli kehrten zwei von uns nach Sankt Petersburg zurück, in die Stadt, wo alles angefangen hatte. Yuri Matiya- sevich hat uns in sein Institut eingeladen, sodass wir ihm unsere neuen Erkenntnisse über die Optimierung diophantischer Gleichungen vorstellen konnten. Zur gleichen Zeit war auch Zhi-Wei Sun zu Besuch und so konnten wir den Mann persönlich kennen lernen, dessen Arbeit wir so gründlich studiert und auch erweitert hatten. Bei dem Besuch machte Matiyasevich uns auch gleich einen neuen Projektvorschlag, diesmal zu Forschung in den Gaußschen ganzen Zahlen.

Das Formalisierungsprojekt hat schon wichtige Teile vollendet, konnte Hilberts 10. Problem jedoch noch nicht vollständig formalisieren. Der bisherige Stand wurde im Juli bei der Federated Logic Conference in Oxford vorgestellt. Im September wird es auch im Rahmen des European Union Contest for Young Scientists (EUCYS) präsentiert, einem Wettbewerb der ähnlich aufgebaut ist wie „Jugend forscht".

\section{Danksagung}

Wir danken unserem Projektbetreuer Dierk Schleicher, der uns immer unterstützt und Kontakte vermittelt hat. Wir danken Yuri Matiyasevich, der uns ermutigt hat, sein Theorem formal zu verifizieren und den universellen Paaren über den ganzen Zahlen nachzugehen. Zu guter Letzt danken wir unseren Freunden an der Jacobs University Bremen, die tatkräftig am Formalisierungsprojekt mitgewirkt haben.

Der zweite Beitrag stammt von Michael Schmalian (18), Abiturient des Helmholtz-Gymnasium in Karlsruhe, der von Frank Herrlich (Karlsruhe) betreut wurde. Er erhielt den 2. Preis im Fachgebiet Mathematik/Informatik (gestiftet von der Fraunhofer-Gesellschaft zur Förderung der angewandten Forschung e. V). und zusätzlich den Preis für eine außergewöhnliche mathematische Arbeit (gestiftet von der DMV).

Zylinder auf der Chamanara-Fläche

\section{Einleitung}

Ausgangspunkt dieses Projekts ist das bereits ausführlich behandelte Konzept der Translationsflächen (s. Abschnitt zu Grundlagen für Definitionen oder [3] für grundlegende Resultate). Grob gesagt ist eine Translationsfläche ein Vieleck dessen Seiten miteinander „verklebt“ wurden. Meistens wird von endlich vielen Verklebungen ausgegangen, doch einige neue Resultate betrachten unendlich viele solcher Verklebungen. Durch diese Verallgemeinerung werden einige kanonische Resultate ungültig, womit auch ein Verlust an Intuition einhergeht. Um sich dieser Schwierigkeit zu nähern hat Reza Chamanara in [2] eine besonders symmetrische unendliche Translationsfläche vorgeschlagen.

Eine Intuition für jene Chamanara-Fläche zu schaffen steht im Mittelpunkt dieses Projekts. Wir haben beobachtet, dass klassische Objekte, der Verlauf von Strahlen oder genauer Zylindern, auf der Chamanara-Fläche sehr symmetrische Erscheinungsformen besitzt. Um dies zu beweisen, mussten wir eine formale algebraische Be- schreibung der gegebenen Objekte schaffen. Durch diese Beschreibung wurden unsere Symmetrien zu Korollaren von Resultaten aus [1]. Relativ wenige Teile der Beweise nutzen spezifische Eigenschaften der Chamanara-Fläche, sodass unsere Resultate und Intuition auf andere Flächen angewandt werden können. Im Grunde probieren wir hier besser zu verstehen, wie wir algebraische Beschreibung schnell auf geometrische Eigenschaften abbilden können.

\section{Grundlagen}

Eine Fläche, die durch Verkleben paralleler gleich langer (also durch Translationen ineinander überführbarer) Seiten von Polyedern entsteht, nennen wir Translationsfläche. Beispielhaft ist ein Rechteck, dessen gegenüberliegende Seiten verklebt wurden, ein Torus (s. Abb. 1). Wir betrachten ein Einheitsquadrat und unterteilen jede Seite in eine Folge von Abschnitten $a[1], a[2], \ldots$ mit Häufungspunkten in gegenüberliegenden Ecken, sodass die Länge von $a^{[i]}$ gleich $0.5^{i}$ ist. Das Identifizieren gegenüberliegender 

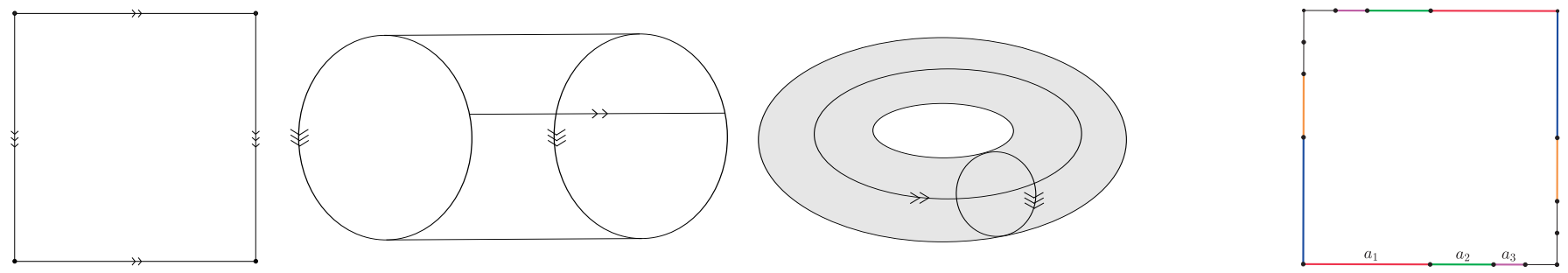

Abbildung 1. Links: Torus als Translationsfläche, rechts: Chamanarafläche

gleich langer Abschnitte bildet die Chamanara-Fläche $X$ (s. Abb. 1).

Wenn wir eine Translationsfläche verkleben, bezeichnen wir die Endpunkte der verklebten Strecken als Singularitäten. Der Verlauf eines Strahls auf einer Translationsfläche ist eine Geodätische. Eine Geodätische ist also ein zusammenhängender Verlauf, der lokal geradlinig ist und an verklebten Stellen seine Richtung beibehält (s. Abb. 2).

Eine Menge paralleler, benachbarter, geschlossener und gleich langer Geodätischen, nennen wir einen Zylinder. Der Abstand zwischen den Rändern eines Zylinders ist seine Höhe. Die Länge seiner Geodätischen ist sein Umfang. Der Quotient aus Umfang und Höhe ist sein Modul. Die bis auf Ränder vollständige Zerteilung einer Fläche in maximale (unter der TeilmengenOrdnungsrelation) Zylinder, bezeichnen wir als Zylinderzerlegung (s. Abb. 2).

Veech-Gruppe: Eine Selbstabbildung einer Fläche heißt genau dann lokal affin, wenn jeder Punkt in einer Umgebung liegt, die bis auf die „Verklebungen“ bzw. Translationskarten affin abgebildet wird. Der lineare Anteil beziehungsweise die Matrix einer lokal affinen Abbildung ist hier eindeutig. Somit bezeichnen wir die Untergruppe von $S L(2, \mathbb{R})$ der Matrizen aller lokal affiner und orientierungserhaltenden Abbildungen einer Translationsfläche als ihre Veech-Gruppe $\Gamma$.

\section{Resultat}

Jede Zylinderzerlegung der Chamanara-Fläche hat zwar unendliche viele Zylinder, doch unter diesen kommen höchstens zwei verschiedene Zylindermoduln vor.

\section{Beweisskizze}

Wir wollen erstmal verstehen, wie Zylinder mit Elementen der Veech-Gruppe zusammenhängen. Affine Abbildungen deren Matrizen Spur 2 haben, wirken als Scherungen. Solche Abbildungen nennen wir parabolisch. Wirkt eine solche Scherung in die gleiche Richtung wie eine Zylinderzerlegung, so bleiben alle Zylinder erhalten (s. Abb. 2). Damit also eine solche Matrix in der VeechGruppe liegt muss die entsprechende lokal affine Abbildung auf der gesamten Translationsfläche nur noch stetig sein. Dies ist erfüllt, wenn die Ränder der Geodätischen jedes Zylinders punktweise erhalten bleiben beziehungsweise die einzelnen Zylinderscherungen an ihren Rändern zusammenpassen. Der Rand bleibt genau dann erhalten, wenn er um ein Vielfaches des Zylinderumfangs geschert wurde und damit ganzzahlig oft „um den Zylinder rumgelaufen ist“. Dazu muss das Inverse des Zylindermoduls ein ganzzahliges Vielfaches der Steigung der Scherung sein. Damit also Stetigkeit für alle Zylinder erfüllt ist, muss eine Zahl existieren, die ein natürliches Vielfaches aller Zylindermoduln ist.

Unterteilt man die Chamanara-Fläche $X$ in zwei Bereiche $A$ und $B$ (s. Abb. 3 ), so gibt es in $A$ nur endlich viele Zylinder. Alle Zylinder die nur in $B$ liegen, sind durch Strahlensätze zu einander ähnlich und haben somit den gleichen Modul. Somit gibt es endlich viele verschiedene Zylindermoduln. Man kann den Verlauf jeder Geodätischen modellieren durch Aneinanderkleben mehrerer Kopien von $X$ (s. Abb. 3). Es wird klar, dass die Endpunkt der Geodätischen rationale Koordinaten haben. Da alle Zylinder die gleiche Richtung und rationale Koordinaten haben, werden alle Zylindermoduln ein Produkt aus einer
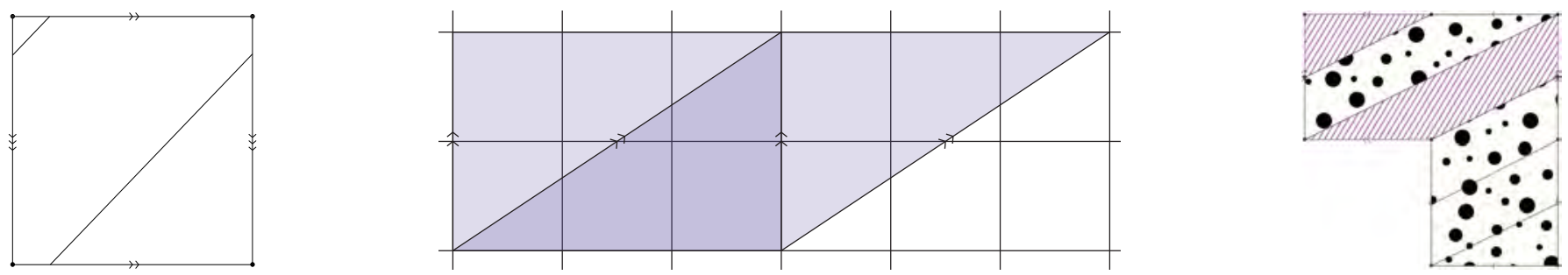

Abbildung 2. Links: Geodätische auf dem Torus, Mitte: Zylinderscherung, rechts: Zylinderzerlegung 

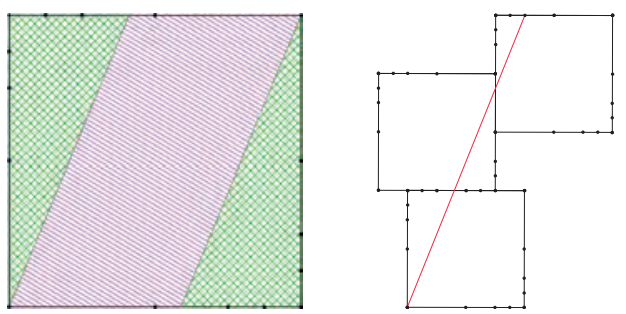

Abbildung 3. Links: Unterteilung in A und B, rechts: Geodätisch auf X
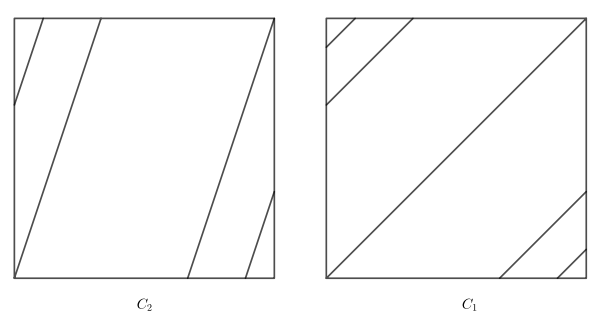

Abbildung 4. Links $\mathrm{C}_{1}$, rechts $\mathrm{C}_{2}$ gemeinsamen, von der Richtung abhängigen konstanten und einer rationalen Zahl sein. Somit existiert auch eine Zahl, die ein natürliches Vielfaches aller Zylindermoduln ist und es bedeutet auch, dass zu jeder Zylinderzerlegung ein parabolisches Element in der Veech-Gruppe liegt.

Wir nutzen jetzt einige Resultate aus der Arbeit [1]. Hier wird die Veech-Gruppe der Chamanara-Fläche genau beschrieben. Als Korollar wird dort bewiesen, dass jedes parabolische Element in der Veech-Gruppe innerhalb dieser Gruppe zu einer ganzzahligen Potenz einer der folgenden beiden Elemente ähnlich ist:

$$
P_{1}=\left(\begin{array}{ll}
1 & 6 \\
0 & 1
\end{array}\right), \quad P_{2}=\frac{1}{4} \cdot\left(\begin{array}{ll}
-5 & 27 \\
-3 & 13
\end{array}\right) .
$$

Diese beiden Matrizen entsprechen zwei sehr einfachen Zylinderzerlegungen $C_{1}$ und $C_{2}$ :

Jede beliebige Zylinderzerlegung $C$ von $X$ besitzt ein parabolisches Element $\delta \in \Gamma$. Nach obiger Aussage in [1] ist o. B. d. A. $\delta=\gamma \cdot P_{1}^{n} \cdot \gamma^{-1}$. Es gilt $\delta\left(\gamma\left(C_{1}\right)\right)=$ $\gamma \cdot P_{1}^{n} \cdot \gamma^{-1}\left(\gamma\left(C_{1}\right)\right)=\gamma\left(C_{1}\right), \gamma \in \Gamma$. Elemente der VeechGruppe erhalten Linien, Zylinder und damit auch Zylinderzerlegungen. Zusätzlich wird in jede Richtung um das gleiche Maß gestreckt, sodass die Anzahl der Zylindermoduln unverändert bleibt. Somit ist $\gamma\left(C_{1}\right)$ die Zylinderzerlegung zu $\delta$ und damit gleich $C$. $C$ hat damit gleich viele Zylindermoduln wie $C_{1}$ oder $C_{2}$. Durch Betrachtung dieser beiden einfachen Zylinderzerlegungen sehen wir, dass sie jeweils eine oder zwei verschiedene Zylindermoduln besitzen und damit das Theorem bewiesen ist.

Die Definition einer Chamanara-Fläche kann man mittels eines Parameters $x \in(0,1)$ verallgemeinern. Die zu verklebenden Abschnitte $a[i]$ besitzen dabei die Länge $x^{i}$. Wir haben bisher mit $x=0.5$ gearbeitet. Wir konnten für diese allgemeinere Definition für alle Parameter der Form $x=\frac{1}{n}, n \in \mathbb{N}$ Resultate aus [1] fortsetzen und damit Postulate aus [2] bestätigen.

\section{Danksagung}

Professor Frank Herrlich hat mein Interesse für dieses Thema geweckt und mir geholfen die notwendigen Grundlagen zu verstehen. Ich bedanke mich ganz herzlich dafür!

\section{Literatur}

[1] Frank Herrlich, Anja Randecker, Notes on the Veech group of the Chamanara surface, 2016, preprint. arxiv.org/pdf/1612. o6877.pdf.

[2] Reza Chamanara, Affine automorphism groups of surfaces of infinite type. In: The Tradition of Ahlfors and Bers, III (William Abikoff and Andrew Haas, eds.), Contemporary mathematics, vol. 335, 2004, 123-145.

[3] William A. Veech, Teichmüller curves in moduli space, Eisenstein series and an application to triangular billiards. Inventiones Mathematicae 97 (1989), 553-583.

Kontakt:

Stephanie Schiemann, DMV-Netzwerkbüro Schule-Hochschule,

Freie Universität, Institut für Mathematik, Arnimallee 7, 14195 Berlin

schiemann@math.fu-berlin.de

Jonas Bayer (18) nahm 2016 am International Tournament of Young Mathematicians, einem Mathematikwettbewerb, in dem die Diskussion im Mittelpunkt steht, teil. Später erhielt er ein Stipendium für die Jacobs University in Bremen, an der er nun Mathematik im dritten Semester studiert. Im Sommer 2018 reiste er, wie auch Malte, nach Sankt Petersburg und stellte Yuri Matiyasevich das gemeinsame Optimierungsprojekt vor, welches zuvor auch einen 3. Preis im Bundeswettbewerb Jugend forscht erhielt.

Marco David (17) präsentierte das Formalisierungsprojekt „Hilbert meets Isabelle“ bei einer Konferenz für computergestütztes Beweisen in Oxford. Er studiert im dritten Semester Physik an der Jacobs University Bremen.

Simon Dubischar (17) qualifizierte sich für die Bundesrunde der Mathematik-Olympiade und erreichte die dritte Runde des Bundeswettbewerbs Mathematik, wo er letztes Jahr im Kolloquium die Teilnahme an der Deutschen Schülerakademie gewonnen hat. Gefördert wurde er außerdem noch vom Verein „Mathe in Bremen e. V.“. Im kommenden Jahr wird er seine schulische Laufbahn am Kippenberg Gymnasium in Bremen abschließen. 


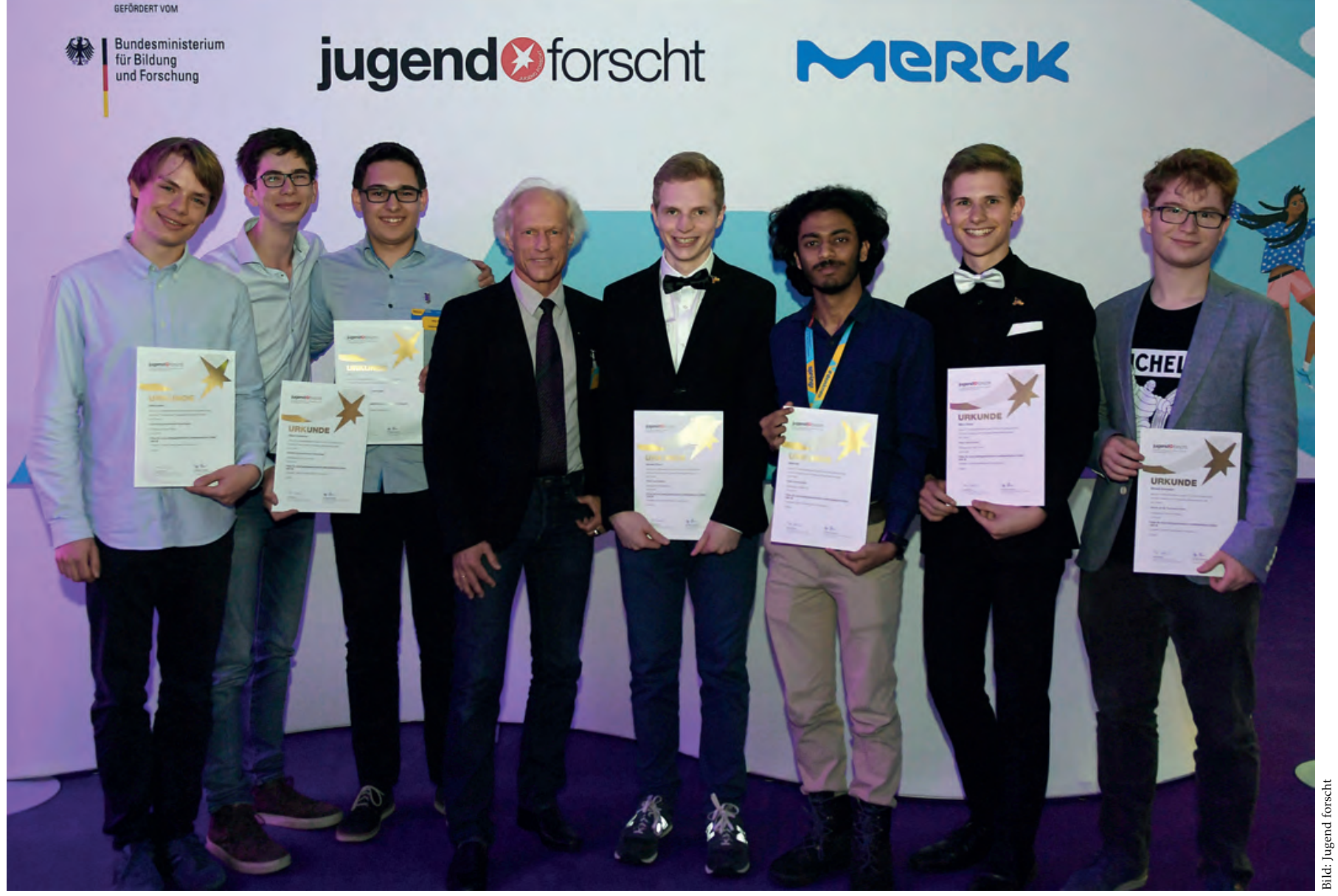

Die Preisträger des DMV-Sonderpreises bei ,Jugend forscht' mit dem Präsidenten der DMV, Michael Röckner: Malte Haßler, Simon Dubischar, Jonas Bayer, Michael Röckner, Benedikt Stock, Abhik Pal, Marco David und Michael Schmalian (v. 1.n. r.)

Malte Haßler (18) nahm in den Jahren 2015 und 2016 am International Tournament of Young Mathematicians teil. Des Weiteren qualifizierte er sich dreimal für die Bundesrunde der Mathematik Olympiade und war, zusammen mit Jonas Bayer und Simon Dubischar, zweimal Preisträger beim Bundeswettbewerb von Jugend forscht. Auch er studiert zurzeit mit der

Unterstützung eines Stipendiums im dritten Semester Mathematik an der Jacobs University Bremen.

Abhik Pal (19) ist aus Indien und hat dort seinen Schulabschluss gemacht, bevor er 2017 als Student an die Jacobs University Bremen kam. Dort studiert er Mathematik mit Nebenfach Informatik. Er war studentischer Entwickler für den Google Summer of Code 2017 und 2018. Zudem ist er Maintainer einer Open-Source-Visualisierungsbibliothek.

Stephanie Schiemann (52) leitet das DMV-Netzwerkbüro Schule-Hochschule seit 2010. Sie war Mathematik- und Sportlehrerin, war viele Jahre Schulbuchautorin, in der Lehrerfortbildung tätig und leitete über 25 Jahre Schüler-Talentfördergruppen in Hamburg und Niedersachsen. Seit 2010 ist sie für den Schülerwettbewerb „Mathe im Advent" (www.mathe-im-advent.de) zuständig und hat im Jahr 2016 für dessen Ausrichtung gemeinsam mit Robert Wöstenfeld die gemeinnützige GmbH Mathe im Leben gegründet.

Michael Schmalian (18) stammt aus einer mathematisch geprägten Familie und habe von Jung auf sehr viel Spaß an diesem Fach gehabt. Deshalb nahm er mehrmals an der Deutschen Mathematik Olympiade und dem Bundeswettbewerb Mathematik teil. Er hatte das Glück, zwei Bundessiege im letzteren dieser Wettbewerbe zu erhalten und wurde so für einen Monat an das Max-Planck-Institut für Mathematik nach Bonn eingeladen, wo er an Spezialfällen von Manins Vermutung arbeiten konnte.

Neben einem Schülerstudium am Karlsruher Institut für Technologie wurde er gefördert von dem in Baden ansässigem Hector-Seminar. Dieses nahm mit Prof. Herrlich des KIT Kontakt auf, woraus diese Arbeit entstand. Er plant im nächsten Semester ein Mathematik Studium zu beginnen.

Benedikt Stock (19) war ab der 9. Klasse für zwei Jahre Juniorstudent der Mathematik an der Universität Hamburg. Daneben erreichte er den 1. Preis in der zweiten Runde des Bundeswettbewerbs Mathematik sowie die Bundesrunde der

Mathematik-Olympiade. Beim Bundeswettbewerb erhielt er in der dritten Runde einen Sonderpreis der DMV. Nach der

Teilnahme an der „International Summer School for Students - Modern Mathematics“ erhielt er ein Stipendium an der Jacobs University Bremen, wo er zurzeit im dritten Semester Mathematik studiert.

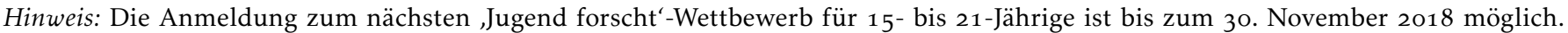
Informationen: www.jugend-forscht.de/teilnahme/alterssparten/jugend-forscht.html. 